\title{
Neues zur Therapie motorischer L-Dopa-Komplikationen
}

\author{
Motorische Komplikationen sind der Preis, den nahe- \\ zu alle Parkinson-Patienten im Laufe ihrer Erkrankung \\ für eine Behandlung mit L-Dopa oder Dopaminago- \\ nisten bezahlen. Der Bedarf an symptomatischen \\ Therapien ist bislang ungedeckt. In den letzten \\ Jahren wurden mehrere Studien zu medikamentösen \\ Strategien für die Behandlung dieser schwerwiegen- \\ den Komplikationen publiziert.
}

Auch wenn es zahlreiche dopaminerge und nicht dopaminerge Therapien für die belastenden motorischen Symptome gibt, sind Wirkungsfluktuationen, Hyper- und Dyskinesien sowie Dystonien laut Professor Jörg B. Schulz, Aachen, nach wie vor nicht beherrschbare Komplikationen und Behandlungslimitationen. L-Dopa-retard-Formulierungen haben die Erwartungen an eine kontinuierliche Stimulation der Dopamin-Rezeptoren bislang nicht erfüllt. Eine gute Erweiterung des Behandlungsinstrumentariums sieht der Experte in einer neuen Kapselformulierung aus L-Dopa und Carbidopa mit zunächst rascher, aber dann lang anhaltender Freisetzung der Substanzen (IPX066Kapseln). Die von der US-amerikanischen FDA bereits im Januar und der europäischen Zulassungsbehörde EMA dann im November 2015 zugelassenen Kapseln können in vier verschiedenen Dosierungen eingesetzt werden. In klinischen Studien wurden sie unter dem Code-Namen IPX066 untersucht. In der bereits 2013 veröffentlichten IPX066-ADVANCE-PD-Studie [Hauser RA et al. Lancet Neurol 2013; 12: 346-56] waren 471 Patienten rekrutiert und zunächst Open-label drei Wochen lang zur individuellen Dosisfindung mit Carbidopa-Levodopa-Immediate Release (CL-IR) sowie im Anschluss sechs Wochen lang ebenfalls Open-label mit IPX066 therapiert worden. 393 Patienten erhielten dann doppelblind randomisiert über 13 Wochen entweder IPX066 oder CL-IR in der individualisierten Dosis. Unter IPX066 war die Zeit in Unbeweglichkeit beziehungsweise im „Off" um 1,17 Stunden kürzer als unter CL-IR und die durchschnittliche Zeit mit guter Beweglichkeit („On“-Zeit) um mehr als eine Stunde länger, hob Schulz hervor. In einer weiteren Studie schnitt IPX066 hinsichtlich der primären und sekundären Endpunkte besser ab als der Komparator. Diesmal wurde IPX066 versus Carbidopa-L-Dopa-Entacapone im CrossoverDesign geprüft und nur Patienten mit motorischen Fluktuationen unter einer stabilen Therapie mit Carbidopa-L-Dopa-Entacapone eingeschlossen [Stocchi F et al. Parkinsonism Relat Disord 2014; 20: 1335 - 40].

Die Randomisierung wurde nach einer Eindosierungsphase mit IPX066 (End of Dose Conversion) vorgenommen. Als primärer Endpunkt war die prozentuale „Off“-Zeit während wacher Stunden am Tag definiert, sekundäre Endpunkte waren die durchschnittliche „Off“-Zeit sowie die durchschnittliche „On“Zeit am Tag ohne beeinträchtigende Dyskinesien. Ausgewertet wurden nur die Daten der Patienten, die die Studie beendet und nach Vorgaben Tagebuch geführt hatten $(n=83)$.

In allen Endpunkten war IPX066 signifikant überlegen: Die prozentuale Zeit im „Off“ war mit $24 \%$ versus $32,5 \%$ signifikant kürzer ( $<<0,0001)$, ebenso wie die „Off“-Zeit am Tag mit 3,8 versus 5,2 Stunden $(p<0,0001)$. Dabei hatten die Patienten unter IPX066 mit 11,4 versus 10,0 Stunden $(\mathrm{p}<0,0001)$ eine längere „On“-Zeit am Tag ohne beeinträchtigende Dyskinesien. „Die Ergebnisse sprechen für sich“, so Schulz. „Bleibt zu hoffen, dass sie dem G-BA ausreichen, um der Formulierung IPX066 einen Zusatznutzen auszusprechen."

Ute Ayazpoor, freie Medizinjournalistin

Bewegungsstörungen (Jörg B. Schulz), 8. Neurologie-Update-Seminar, 19. - 20.2.2016 in Mainz

\section{Schmerz bei Demenz: Hoher Forschungsbedarf}

\begin{abstract}
Menschen mit demenziellen Symptomen können Schmerz häufig nicht mehr verbal kommunizieren. Das heißt aber keinesfalls, dass sie nicht daran leiden. Bisher gibt es nur wenige valide Studien, die sich mit dem täglichen, meist unterrepräsentierten Symptom "Schmerz" bei Demenz befassen.
\end{abstract}

Zwei seines Erachtens hochaktuelle Arbeiten stellte Professor Arne May, Hamburg, beim Neuro Update vor. Die Herausforderung im klinischen Alltag besteht laut May darin, Schmerz bei Menschen mit kognitiven Einschränkungen nicht nur zu erkennen, sondern auch effektiv zu behandeln - und dabei zu berücksichtigen, dass diese Behandlung die Kognition weiter verschlechtern kann. Eine 2015 erschienene Übersichtsarbeit stellt die aktuelle Situation dar: Sie fasste Studien zum Schmerzerleben von Menschen mit verschiedenen Demenzformen zusam- men und diskutierte die wenigen bestehenden Tiermodelle. Die Autoren arbeiteten heraus, dass die Auffassung, demente Patienten seien durch ihre Demenz vor Schmerzen „geschützt", auf der Basis sehr früher und wenig aussagekräftiger Untersuchungen entstand [Defrin R et al., Pain 2015; 156: 1396 - 1408]. Zudem ignorierten die Studien, so die Autoren des Reviews, die inzwischen bekannte Tatsache, dass Schmerzerleben nicht gleichbedeutend mit Schmerzkommunikation ist. Aktuelle Forschungsergebnisse mit neuen Untersuchungsverfahren deuten sogar darauf hin, dass Menschen mit Demenz eine vermehrte Hyperalgesie auf Schmerzreize entwickeln [Hugenschmidt CE et al., Pain 2015; 156: 1396 - 1408]. Der Ursache müsse dringend in weiteren, ,gut gemachten“ Studien nachgegangen werden, kommentierte May. Ute Ayazpoor, freie Medizinjournalistin

Schmerz/Kopfschmerz (Arne May), 8. Neurologie-Update-Seminar, 19. - 20.2.2016 in Mainz 